\title{
INVERSIÓN EXTRANJERA DIRECTA EN MÉXICO: COMPARACIÓN ENTRE LA INVERSIÓN PROCEDENTE DE LOS ESTADOS UNIDOS Y DEL RESTO DEL MUNDO
}

\author{
Ana Carolina Garriga
}

\section{INTRODUCCIÓN}

¿Hay diferencias entre la inversión extranjera directa (IED) que México ha recibido de los Estados Unidos (EE.Uu.) y del resto del mundo? En este trabajo se identifican las características que distinguen la IED recibida por México procedente de los EE.UU. de la IED recibida del resto del mundo. El análisis se realiza no sólo a nivel federal, en términos de IED hacia el país en su conjunto, sino que también se identifican diferencias en la localización de la inversión en las entidades estatales.

La IED procedente de los EE.UU. representa el $46 \%$ de la IED que México recibe. Identificar particularidades en flujos de inversión de distinto origen es importante no sólo como parte de una mejor comprensión de las características de la IED, sino también por dos consecuencias prácticas inmediatas. En primer lugar, esta particularización puede identificar vulnerabilidades en las economías locales y, en segundo, puede informar la toma de decisiones destinadas a la promoción de inversiones tanto a nivel federal, como estatal.

La gráfica 1 muestra la importancia proporcional de la IED procedente de los EE.UU. en el marco de la IED que México ha recibido desde 1999. La magnitud de la IED estadounidense y la distancia que separa la IED de este origen de la IED de los Países Bajos o de España, justifican el interés que motiva este trabajo. 


\section{GRÁFICA 1}

IED total según origen, en dólares corrientes (1999-2015*)

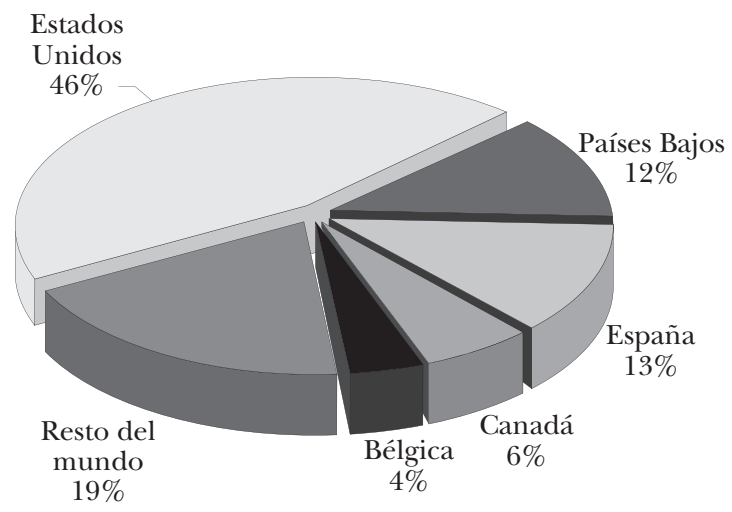

Fuente: Elaboración propia con datos de la Dirección General de Inversión Extranjera, de la Secretaría de Economía.

* Las cifras correspondientes a 2015 sólo reflejan los tres primeros trimestres del año.

\subsection{La IED y su importancia}

Hablamos de IED cuando una persona física o moral extranjera construye una planta o fábrica, o adquiere un emprendimiento ya existente en el país. La Secretaría de Economía de México considera que IED también constituye la compra de por lo menos $10 \%$ de las acciones con poder de voto de sociedades mexicanas ya en funcionamiento o por constituirse. ${ }^{1}$ Según datos oficiales, entre 1999 y el tercer trimestre de 2015, México ha recibido 450, 581 millones de dólares americanos (mdd), de los cuales alrededor del $47 \%$ procedían de los EE.uU. (211, 657 millones de la misma moneda). ${ }^{2}$

Dos simples comparaciones permiten poner en perspectiva la magnitud de los flujos de IED en la economía mexicana. Recuérdese

${ }^{1}$ Secretaría de Economía, "Notas metodológicas sobre IED en México", en http:/ / www.2006-2012.economia.gob.mx/comunidad-negocios/inversion-extranjera-directa/estadistica-oficial-de-ied-en-mexico/notas-metodologicas, consultado el 24.V.2013.

${ }^{2}$ Cifras en dólares constantes de 2012. 
que el producto interno bruto mexicano alcanzó los 1, 295 billones de dólares en 2014, en tanto que las reservas mexicanas cerraron el año 2014 con 195, 681.6 mdd, según cifras del Banco Mundial. ${ }^{3}$ Esto significa que el ingreso promedio anual de divisas por IED en este periodo representa aproximadamente el $2 \%$ del tamaño de la economía y más del $12 \%$ de las reservas del país.

La comprensión de las características y del comportamiento de la IED es especialmente importante para el caso de los países en desarrollo, ya que la IED se ha asociado con una serie de efectos positivos sobre la economía. ${ }^{4}$ Por ejemplo, entre los estudios de agregados nacionales, José Luis de la Cruz y José Antonio NúñezMora han mostrado que hay una relación positiva entre IED y producto interno bruto real, exportaciones e importaciones para el caso de México y que esta relación es unidireccional -la IED mejora estos indicadores, pero no parecen atraer más de ésta. ${ }^{5}$ Gallegos, Canfield Rivera y González Castro llegan a conclusiones semejantes. ${ }^{6}$ Asimismo, también hay evidencia según la cual la IED se asocia con niveles más altos de inversión doméstica. ${ }^{7}$ En lo que toca a las

${ }^{3}$ Nótese que en las estadísticas del Banco Mundial, el total de las reservas incluye no sólo la tenencia de divisas en Banxico, sino también oro monetario, derechos especiales de giro y reservas en el FMI. Véase Banco Mundial, en http:// datos.bancomundial.org/pais/mexico, consultado el 11.XII.2015.

${ }^{4} \mathrm{Al}$ respecto, véase Xiaoying Li y Xiaming Liu, "Foreign Direct Investment and Economic Growth: An Increasingly Endogenous Relationship” World Development, vol. 33, núm. 3, 2005, pp. 393-407, y Theodore H. Moran, Beyond Sweatshops, Washington, D.C., Brookings Institution Press, 2002.

${ }^{5}$ Esto sugiere que la IED en México estaría motivada más por factores externos que por la evolución de la economía nacional. Véase el estudio de J. L. de la Cruz y J. A. Núñez-Mora, "Comercio internacional, crecimiento económico e inversión extranjera directa: evidencias de causalidad para México”, Revista de Economía Mundial, núm. 15, 2006, pp. 181-202.

${ }^{6}$ J. L. de la Cruz Gallegos, Carlos Canfield Rivera y Priscilla González Castro, "Economic Growth, Foreign Direct Investment And International Trade: Evidence on Causality in the Mexican Economy", Revista Brasileira de Economia de Empresas, vol. 9, núm. 1, 2009, pp. 17-29. Este artículo destaca que la actividad económica de los EE.UU. parece tener un impacto más importante sobre la IED que recibe México que el de la propia actividad económica mexicana.

${ }^{7}$ Véase Eduardo Borensztein, José de Gregorio y Jong-Wha Lee, "How Does Foreign Direct Investment Affect Economic Growth?”, Journal of International Eco- 
industrias, varios estudios han encontrado que empresas de capital extranjero generan externalidades positivas sobre las empresas mexicanas. ${ }^{8}$

El impacto de la IED no se limita a variables económicas. Otros estudios han encontrado que la IED también se relaciona con mejoras en los niveles de desigualdad del ingreso en México. ${ }^{9}$ Aún más: en el plano político subnacional, hay evidencia de que la IED fortalece políticamente a los líderes regionales. ${ }^{10}$

Un conocimiento pormenorizado de los flujos de IED que recibe el país es importante para mejores diseño y evaluación de políticas públicas. La atracción de IED forma parte de la agenda de los gobiernos y el mexicano no es una excepción. Además de las políticas domésticas que afectan la IED, independientemente de su origen (tal es el caso, por ejemplo, de la Ley de Inversión Extranjera), México ha tejido una red de acuerdos internacionales para facilitar a otros mercados el acceso de bienes y servicios producidos en el país.

nomics, vol. 45, núm. 1, 1998, pp. 115-135, y Ashoka Mody y Antu Panini Murshid, "Growing Up with Capital Flows", Journal of International Economics, vol. 65, núm. 1, 2005, pp. 249-266.

${ }^{8}$ Véanse, por ejemplo, Brian Aitken, Gordon H. Hanson y Ann E. Harrison, "Spillovers, Foreign Investment, and Export Behavior", Journal of International Economics 43, núms. 1-2, 1997, pp. 103-132; Jacob A. Jordaan, "Determinants of FDI-Induced Externalities: New Empirical Evidence for Mexican Manufacturing Industries", World Development, vol. 33, núm. 12, 2005, pp. 2103-2118, e "Intra- and Inter-industry Externalities from Foreign Direct Investment in the Mexican Manufacturing Sector: New Evidence from Mexican Regions", World Development, vol. 36, núm. 12, 2008, pp. 2838-2854; Chong-Sup Kim, "Los efectos de la apertura comercial y de la inversión extranjera directa en la productividad del sector manufacturero mexicano”, El Trimestre Económico, vol. 64, núm. 255, 1997, pp. 365-390; Miguel D. Ramirez, "Are Foreign and Public Capital Productive in the Mexican Case? A Panel Unit Root and Panel Cointegration Analysis”, Eastern Economic Journal, vol. 36, núm. 1, 2010, pp. 70-87; y David Romo, "Derramas tecnológicas de la inversión extranjera en la industria mexicana”, Comercio Exterior, vol. 53, núm. 3, 2003, pp. 230-243.

${ }^{9}$ Nathan M. Jensen y Guillermo Rosas, "Foreign Direct Investment and Income Inequality in Mexico, 1990-2000”, International Organization, vol. 61, núm. 3, 2007, pp. 467-487.

${ }^{10}$ Edmund J. Malesky, "Straight Ahead on Red: How Foreign Direct Investment Empowers Subnational Leaders", The Journal of Politics, vol. 70, núm. 1, 2008, pp. $97-119$. 
Entre estos acuerdos descuella el Tratado de Libre Comercio de América del Norte (TlCAN) como uno de los más importantes. Sin embargo, México también ha negociado tratados para ofrecer garantías adicionales a la IED. Según la Secretaría de Relaciones Exteriores, hasta el 30 de julio de 2014 México firmó y ratificó veintinueve acuerdos bilaterales de inversión. De éstos, sólo tres (con Argentina, los Países Bajos y Suiza) fueron ratificados hacia finales de los años noventa, mientras que el resto responde a una política activa, puesta en marcha en la primera década del $2000 .{ }^{11}$ Nótese que las relaciones de IED con los EE.UU. no se han regido por un acuerdo bilateral de inversiones específico, sino por el capítulo XI del TLCAN. ${ }^{12}$

Identificar diferencias en la IED según su origen es un primer paso que dar en la comprensión de las determinantes de la IED y en la toma de decisiones destinadas a la promoción de inversiones. En lo que atañe a la IED procedente de los EE.UU., cabe señalar que la bibliografía a propósito sugiere que hay diferencias entre el comportamiento de ésta y la de otra procedencia. Si este dato se conjuga con el hecho de que, en promedio, la IED estadounidense representa alrededor de la mitad de la IED recibida por México entre los años 1999 y 2015, la importancia de caracterizar esta inversión en el caso de México se pone en evidencia. Por esta razón, es de atender la falta de análisis de las características de la IED procedente de los EE.UU. en México, vacío que este artículo pretende colmar en parte.

El resto de este artículo se organiza de la siguiente manera. La segunda sección analiza la bibliografía especializada sobre los determinantes y características de la IED enderezada a México, así como los estudios que sugieren que la IED procedente de los EE. UU. tiene características particulares. La tercera describe las características de la IED que México ha recibido entre 1999 y 2014 y

11 Este número es relativamente bajo si se lo compara con los 39 acuerdos bilaterales de inversión que Chile ha firmado y ratificado, o con los 57 ratificados por Argentina. Sin embargo, evidencia una política más activa que la que llevó a Brasil a ratificar 14 tratados de este tipo o la que llevó a Colombia a establecer este tipo de mecanismos con sólo siete países.

12 Teresa Gutiérrez-Haces, "La inversión extranjera directa en el TLCAN", Economía UNAM, vol. 1, núm. 3, pp. 30-52. 
analiza las particularidades de la IED procedente de los EE.UU. en comparación con la IED de otro origen. El análisis se realiza tanto a nivel de agregados nacionales, como de dinámicas subnacionales. La sección se completa con el análisis multivariado de los factores que explican la localización de la IED de distinto origen en los estados mexicanos. La cuarta analiza hasta qué punto la IED procedente de los EE.UU. responde a una lógica particular, a más de extraer algunas implicaciones de política pública.

\section{REVISIÓN DE LA BIBLIOGRAFÍA ESPECIALIZADA}

\subsection{La IED en México a la luz de su bibliografía}

Una parte importante en los estudios de economía política internacional se dedica a analizar qué factores afectan la localización de la IED. La mayoría de los estudios sigue el modelo de propiedad, ubicación e internalización, conocido por sus siglas en inglés como oli (Ownership, Location and Internalization). ${ }^{13}$ Todo un programa de investigación se ha desarrollado en torno a los determinantes de carácter político que influyen en la decisión de los inversionistas de localizarse en un país determinado. ${ }^{14}$ Sin embargo,

${ }^{13}$ John H. Dunning, "Toward an Eclectic Theory of International Production: Some Empirical Tests”, Journal of International Business Studies, vol. 11, núm. 1, 1980, pp. 9-31, y "The Eclectic Paradigm of International Production: A Restatement and Some Possible Extensions", Journal of International Business Studies, vol. 19, núm. 1, 1988, pp. 1-31.

14 Por ejemplo, Glen Biglaiser y Karl R. DeRouen, "Economic Reforms and Inflows of Foreign Direct Investment in Latin America", Latin American Research Review, vol. 41, núm. 1, 2006, pp. 51-75; Glen Biglaiser y Joseph L. Staats, "Do Political Institutions Affect Foreign Direct Investment? A Survey of U.S. Corporations in Latin America”, Political Research Quarterly vol. 63, núm. 3, 2010, pp. 508-522; Tim Büthe y Helen V. Milner, "The Politics of Foreign Direct Investment into Developing Countries: Increasing FDI through International Trade Agreements?”, American Journal of Political Science, vol. 52, núm. 4, 2008, pp. 741-762; Nathan M. Jensen, Nation-States and the Multinational Corporation. A Political Economy of Foreign Direct Investment, Princeton, NJ, Princeton University Press, 2006; Quan Li, "Democracy, Autocracy, and Tax Incentives to Foreign Direct Investors: A Cross-National 
los estudios referidos a la distribución subnacional de la IED, esto es en qué partes de un país deciden ubicarse las inversiones, son más recientes y menos extendidos. ${ }^{15}$

El estudio de la IED en México ha recibido un renovado interés en la bibliografía académica. Por ejemplo, Mollick, Ramos-Duran y Silva-Ochoa estudian los flujos de IED a los 22 estados mexicanos que recibieron mayor IED en el periodo bajo estudio (entre 1994 y 2001). ${ }^{16} \mathrm{El}$ análisis estadístico identifica qué factores atraen IED a los estados estudiados. Por ejemplo, su artículo señala que la "infraestructura internacional” (medida como líneas telefónicas) tiene una influencia mayor que la infraestructura doméstica (medida como carreteras federales y secundarias) en la atracción de IED a distintos estados, mientras que el gasto público no es un determinante significativo de la IED.

Jordaan analiza la distribución subnacional de la IED en México y presenta evidencia sobre una serie de factores que atraen la inversión a los estados mexicanos: el tamaño del mercado estatal, el nivel de salarios, los años de escolaridad promedio de la población, la infraestructura de comunicaciones y las economías de aglomeración. ${ }^{17}$ Asimismo, muestra diferencias significativas en los factores que determinan la localización de la inversión en maquiladoras,

Analysis”, The Journal of Politics, vol. 68, núm. 1, 2006, pp. 62-74; y Pablo M. Pinto, Partisan Investment in the Global Economy: Why the Left Loves Foreign Direct Investment and FDI Loves the Left, Cambridge, MA, Cambridge University Press, 2013.

${ }^{15}$ Por cuestiones de espacio, se omite el análisis de la "nueva" bibliografía que estudia la distribución subnacional de IED en otras economías en desarrollo. Véanse, por ejemplo, Agnieszka Chidlow, Laura Salciuviene y Stephen Young, "Regional Determinants of Inward FDI Distribution in Poland", International Business Review, vol. 18, núm. 2, 2009, pp. 119-133; Klaus E. Meyer y Hung Vo Nguyen, "Foreign Investment Strategies and Sub-national Institutions in Emerging Markets: Evidence from Vietnam”, Journal of Management Studies, vol. 42, núm. 1, 2005, pp. 63-93; y Megha Mukim y Peter Nunnenkamp, "The Location Choices of Foreign Investors: A District-level Analysis in India”, The World Economy, vol. 35, núm. 7, 2012, pp. 886-918.

${ }^{16}$ Véase su estudio "Infrastructure and FDI Inflows into Mexico: A Panel Data Approach”, Global Economy Journal, vol. 6, núm. 1, 2006, pp. 1-25.

17 "State Characteristics and the Locational Choice of Foreign Direct Investment: Evidence from Regional FDI in Mexico 1989-2006", Growth and Change, vol. 39, núm. 3, 2008, pp. 389-413. 
la cual no responde -como podría esperarse-al tamaño del mercado local ni a mejor infraestructura.

En un trabajo reciente, Samford y Ortega Gómez estudian la IED en México entre los años 2000 y 2009, pero desagregando la inversión, según tenga por fin la explotación de recursos, de mercados o de oportunidades de eficiencia, y estudian sus determinantes en una muestra de todos los estados mexicanos. ${ }^{18}$ Sus resultados indican que al margen de los factores geográficos y económicos que tradicionalmente se asocian con la IED, hay una serie de factores políticos que adquieren relevancia, tales como el control partidario del Estado, la estabilidad social y la eficiencia (percibida) de las autoridades estatales. Asimismo, hay interesantes diferencias que dependen del destino (o tipo) de IED en particular.

Escobar Gamboa analiza la distribución subnacional de la IED en México en el periodo 1994-2004. Sus resultados indican que ésta responde a menor delincuencia y a mayor educación, así como a la presencia de efectos regionales de atracción de inversión. ${ }^{19}$ Garriga, en cambio, realiza un análisis de las determinantes de la IED nueva, entre 1999 y $2012 .{ }^{20}$ Los resultados indican que entre los factores que atraen la IED nueva a los estados mexicanos se encuentran los rangos de IED previos, mayor escolarización de la población y mejores salarios, lo que sugiere una preferencia de los inversionistas por estados con mano de obra más calificada y mejores conexiones por carreteras federales. El análisis también indica que en este periodo, las economías más grandes recibieron proporcionalmente menos IED que las más pequeñas y que el partido político que gobierna el Estado tiene una influencia marcada en los flujos de IED que él mismo recibe. Contrario a las expectativas de parte de la bibliografía especializada (y de los resultados

\footnotetext{
18 "Subnational Politics and Foreign Direct Investment in Mexico", Review of International Political Economy, vol. 22, núm. 2, 2014, pp. 467-496.

19 "Foreign Direct Investment (FDI) Determinants and Spatial Spillovers across Mexico's States”, The Journal of International Trade E Economic Development, vol. 22, núm. 7, 2013, pp. 993-1012.

20 "Política partidaria e inversión extranjera directa. El Caso de los estados mexicanos", documento de trabajo DTEP, núm. 256, México, Centro de Investigación y Docencia Económicas, 2013.
} 
de Samford y Ortega Gómez), pero consistente con el trabajo de Pinto y otros, ${ }^{21}$ los estados gobernados por el PRD reciben mayores flujos de IED que los estados gobernados por el PAN o el PRI.

Garriga y Phillips estudian las consecuencias de la violencia criminal en los flujos de IED entre 2000 y 2012. ${ }^{22}$ Los autores no encuentran una relación directa entre crimen organizado y IED. Sin embargo, este trabajo apunta que la violencia criminal condiciona la relación entre IED y variables, como la democracia, el crecimiento económico y el capital humano. Estos factores sólo atraen inversión, cuando los niveles de violencia criminal son bajos.

Todos estos trabajos no hacen más que poner de manifiesto la complejidad del tema y la diversidad de factores asociados con la atracción de IED, así como la necesidad de contar con diagnósticos adecuados de los flujos de inversión que México recibe. Cabe destacar que la bibliografía citada supone que no hay diferencias según el origen de la IED. En este marco se inscribe el presente estudio, que intenta aportar más elementos a la comprensión de la IED en México, al distinguir las características de la IED procedente de los EE.uU., de la IED de otro origen.

\subsection{Diferencias según el origen de la IED}

Hay un par de artículos que señala algunas diferencias en el comportamiento de la IED procedente de los EE.UU. en relación con la IED procedente de otros países. ${ }^{23}$ Esas diferencias

21 "The Politics of Investment Partisanship and the Sectoral Allocation of Foreign Direct Investment”, Economics Eீ Politics, vol. 20, núm. 2, 2008, pp. 216-254.

22 "Criminal Violence and the Subnational Distribution of Foreign Direct Investment”, documento de trabajo DTEP, núm. 279, México, Centro de Investigación y Docencia Económicas, 2015.

${ }^{23}$ Nigel Driffield y James H. Love, "Linking FDI motivation and host economy productivity effects: Conceptual and empirical analysis", Journal of International Business Studies, vol. 38, núm. 3, 2007, pp. 460-473; y Steven Globerman y Daniel M. Shapiro, "The Impact of Government Policies on Foreign Direct Investment: The Canadian Experience”, Journal of International Business Studies, vol. 30, núm. 3, 1999, pp. 513-532. 
se han documentado en el caso de Asia Oriental. ${ }^{24}$ Algunos atribuyen esas diferencias a la influencia de factores políticos ${ }^{25} \mathrm{o}$ de seguridad. ${ }^{26}$ En el caso de México, que haya diferencias en el comportamiento de la IED de los EE.UU. y la IED del resto del mundo no debería sorprender, dada la especial relación entre México y su vecino del norte, la historia de las relaciones políticas y económicas bilaterales, los diferenciales de costo que impone la cercanía con el mercado estadounidense, la influencia del TLCAN, ${ }^{27}$ la tradición en inversión y la existencia de redes de información que facilitan la IED, ${ }^{28}$ la presencia de migrantes mexicanos en EE.UU., ${ }^{29}$ entre tantos otros factores. Sin embargo, hasta el momento no hay en la bibliografía especializada análisis comparativos del comportamiento de la IED en México según el origen de estos capitales. ${ }^{30}$ Esta información es relevante, ya que puede influir en las estrategias de

${ }^{24}$ Mahani Zainal Abidin y Nor Izzatina Abdul Aziz, "US Foreign Direct Investment in East Asia: Strategy and Policy Issues”, Asian Economic Policy Review, vol. 4, núm. 2, 2009, pp. 248-266, y Mitsuyo Ando, Sven W. Arndt, y Fukunari Kimura, "Production Networks in East Asia: Strategic Behavior by Japanese and US firms", manuscrito en ssRN, http:/ /ssrn.com/abstract=981769 or http:/ / dx.doi.org/10.2 $139 / \operatorname{ssrn} .981769$

${ }^{25}$ Como Glen Biglaiser y David Lektzian, "The Effect of Sanctions on U.S. Foreign Direct Investment”, International Organization, vol. 65, núm. 3, 2011, pp. 531-551.

${ }^{26}$ Véase Glen Biglaiser y Karl R. DeRouen, "Following the Flag: Troop Deployment and U.S. Foreign Direct Investment", International Studies Quarterly, vol. 51, núm. 4, 2007, pp. 835-854.

${ }^{27}$ Patricio Aroca y William F. Maloney, "Migration, Trade, and Foreign Direct Investment in Mexico", The World Bank Economic Review, vol. 19, núm. 3, 2005, pp. 449-472, y Alfredo Cuevas, Miguel Messmacher, y Alejandro Werner, "Foreign Direct Investment in Mexico since the Approval of nafta", The World Bank Economic Review, vol. 19, núm. 3, 2005, pp. 473-488.

${ }^{28}$ M. Ando, S. W. Arndt, y F. Kimura, manuscrito citado.

${ }^{29}$ Beata S. Javorcik et al., "Migrant Networks and Foreign Direct Investment", Journal of Development Economics, vol. 94, núm. 2, 2011, pp. 231-241, y David Leblang, "Familiarity Breeds Investment: Diaspora Networks and International Investment”, American Political Science Review, vol. 104, núm. 3, 2010, pp. 584-600.

${ }^{30}$ Una excepción es el trabajo de Waldkirch ("The Effects of Foreign Direct Investment in Mexico since NAFTA", World Economy, vol. 33, núm. 5, 2010, pp. 710$745)$, quien analiza el efecto distinto de la IED en la productividad y los salarios en los primeros diez años del TLCAN que depende, entre otros factores, del origen de la inversión. 
promoción del desarrollo del gobierno federal y de los gobiernos estatales.

\section{La ied EN MÉxico y Las PARTicularidades DE LA IED ESTADOUNIDENSE}

\subsection{El panorama nacional}

Este artículo se centra en la evolución de la IED en México entre 1999 y 2014. Si bien otros estudios incluyen la década de los noventa en sus muestras, el periodo inmediatamente posterior a la Ley de Inversión Extranjera del 27 de diciembre de 1993 y la firma del TLCAN se excluyen por dos razones. Primero, la inclusión de esos datos introduce el riesgo de reflejar los efectos en el corto plazo de las reformas y las peculiares circunstancias de la política. ${ }^{31}$ En particular, el gran influjo de inversiones de maquila en los estados de la frontera y de inversiones correspondientes a privatizaciones puede haber correspondido a una ventana de oportunidad única que se presentó al liberalizarse la inversión y facilitarse el comercio. Segundo, la Secretaría de Economía alteró la metodología para calcular la IED en 1999, lo cual hace preferible la comparación de datos a partir de ese año. La Secretaría de Economía anunció otro cambio en la metodología de cálculo a partir de 2014, por lo que este trabajo incluye datos nacionales hasta 2014 y datos estatales hasta 2013, el último año completo disponible.

Tres notas metodológicas deben tenerse en cuenta para interpretar la información presentada en este artículo. Primero, hay quienes cuestionan la metodología usada para el registro de la IED hacia los estados mexicanos durante este periodo (y en parte, a esto se debe el cambio en la metodología de registro iniciado en 2014).$^{32}$ En particular, se sospecha que los cuestionarios empleados

31 Jorge M. Márquez Pozos, Alejandro Islas y Francisco Venegas-Martínez, "Corrientes internacionales de capital e inversión extranjera de cartera. El caso de México, 1989-1999”, El Trimestre Económico, vol. 70, núm. 280, 2003, pp. 791-833.

${ }^{32}$ Entrevistas con funcionarios de la Dirección General de Inversión Extranjera. 
llevan a sobre-representar la IED recibida por los estados en los que se encuentran las oficinas principales de las empresas (especialmente, la Ciudad de México). Sin embargo, utilizo estos datos, porque no hay razón para sospechar que hay un sesgo adicional y que el registro de inversión procedente de los EE.UU. sea diferente del registro de fondos procedentes de otros países, que es materia de este trabajo. ${ }^{33}$ Segundo, los datos que utilizo no permiten distinguir posibles procesos de "intermediación" de la IED (por ejemplo, si capitales japoneses invierten en EE.UU. y luego la compañía estadounidense invierte en México). No hay datos que permitan corregir los flujos de IED si se eliminaran estos procesos de intermediación. Hay dos razones para usar datos que potencialmente contienen distorsiones por este tipo de intermediaciones. Por un lado, la bibliografía sugiere que la IED de los EE.uU. comparte algunos rasgos a pesar de posibles movimientos de intermediación. ${ }^{34}$ La existencia de una proporción importante de movimientos de intermediación debería tender a suavizar o hasta eliminar posibles diferencias entre inversionistas, según su origen. En otras palabras, el eventual sesgo que estos movimientos introducen debería operar en contra de los resultados que presento. Tercero, la información que se muestra en esta sección es de índole descriptiva y no incluye ajustes o controles en términos de la situación económica o política, ni de México ni de los distintos países de los que procede la inversión. Sin embargo, esta información sugiere características particulares de la IED procedente de los EE.UU. en relación con la IED global que recibe México.

En este periodo, México recibió al año un promedio de 26, 882 mdd constantes, de los cuales aproximadamente la mitad correspondió a fondos procedentes de los EE.UU. (en promedio, la IED anual procedente de los EE.UU. fue de 12,490 mdd). Este dato agregado, sin embargo, oscurece grandes diferencias en cuanto a los patrones de inversión y tendencias de la IED, según su origen.

\footnotetext{
${ }^{33}$ Sí podría afectar la información presentada en el mapa 1, si llevara a mover algún estado del quintil en que se lo ubicó.

${ }^{34}$ Biglaiser y DeRouen, art. cit.; Biglaiser y Lektzian, art. cit.; y Biglaiser y Staats, art. cit.
} 
Como muestra la gráfica 2 (primer panel), la IED procedente de los Ee.uU. impuso una nueva marca en el año 2001, cuando alcanzó los 25,969 mdd de 2012, seguido por las cifras alcanzadas en los años 2000 y 2002. El peor año para el influjo de capitales procedentes de EE.UU. fue el 2014, cuando sólo ingresaron 7,199 mdd. Este patrón de inversión difiere significativamente de la IED recibida del resto del mundo, que alcanzó marcas sin precedente de IED en los años 2013, ${ }^{35}$ seguidos por el 2010 y el 2014. Aun cuando la crisis financiera global afectó los flujos de IED procedente de otros países, la caída no fue tan grande como en el caso de la IED estadounidense, sin llegar a caer por debajo de los niveles de IED anteriores al año 2000.

La gráfica 2 también refleja diferencias por tipos de flujo. Si bien en el periodo examinado la importancia relativa de los EE.UU. en nuevas inversiones ha caído, este país es el principal responsable de los flujos de reinversión y de cuentas entre compañías (véanse los paneles inferiores de la gráfica 2).

La gráfica 3 muestra la importancia proporcional de la IED procedente de los EE.uU. en el marco de la IED que recibe México. Aun cuando durante el periodo examinado la IED procedente de los EE.UU. representa aproximadamente el $47 \%$ de la IED total, la importancia de la IED de este origen ha variado marcadamente a lo largo de los últimos dieciséis años. Mientras que a principios de la década del 2000 la IED estadounidense llegó a representar más del $70 \%$ de los flujos de IED a México, una mayor diversificación de inversionistas, junto con la caída de las inversiones estadounidenses, llevaron la participación de las inversiones procedentes del norte por debajo del 50\%. Aun cuando el año 2010 fue excepcional, porque se conjugaron una caída de la IED estadounidense y un alto volumen de IED de otro origen, los flujos de inversión estadounidenses recuperaron su importancia relativa en los años siguientes.

${ }^{35}$ En gran medida, producto de la compra del grupo Modelo por la empresa europea AB InBev. Véase http://www.forbes.com.mx/venta-de-grupo-modelo-favorece-inversion-en-mexico/, consultado el 31.V.2016. 


\section{GRÁFICA 2}

IED total y por origen. Distintos flujos, en mdd constantes de 2012
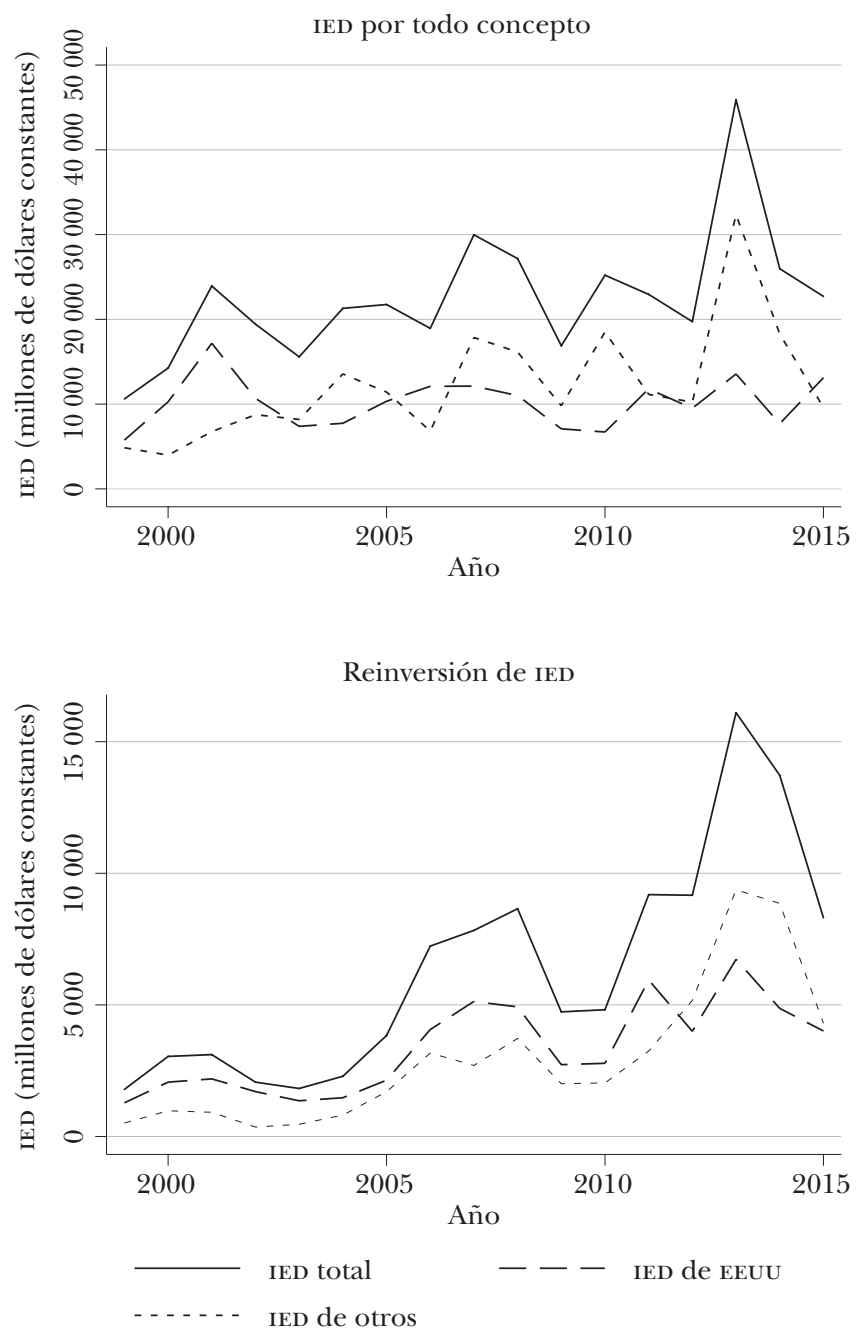

Fuente: Elaboración propia con datos de la Dirección General de Inversión Extranjera, de la Secretaría de Economía.

Nota: Las cifras correspondientes al año 2015 sólo reflejan los tres primeros trimestres del año. 
GRÁfICA 2 (concluye)

IED total y por origen. Distintos flujos, en mdd constantes de 2012
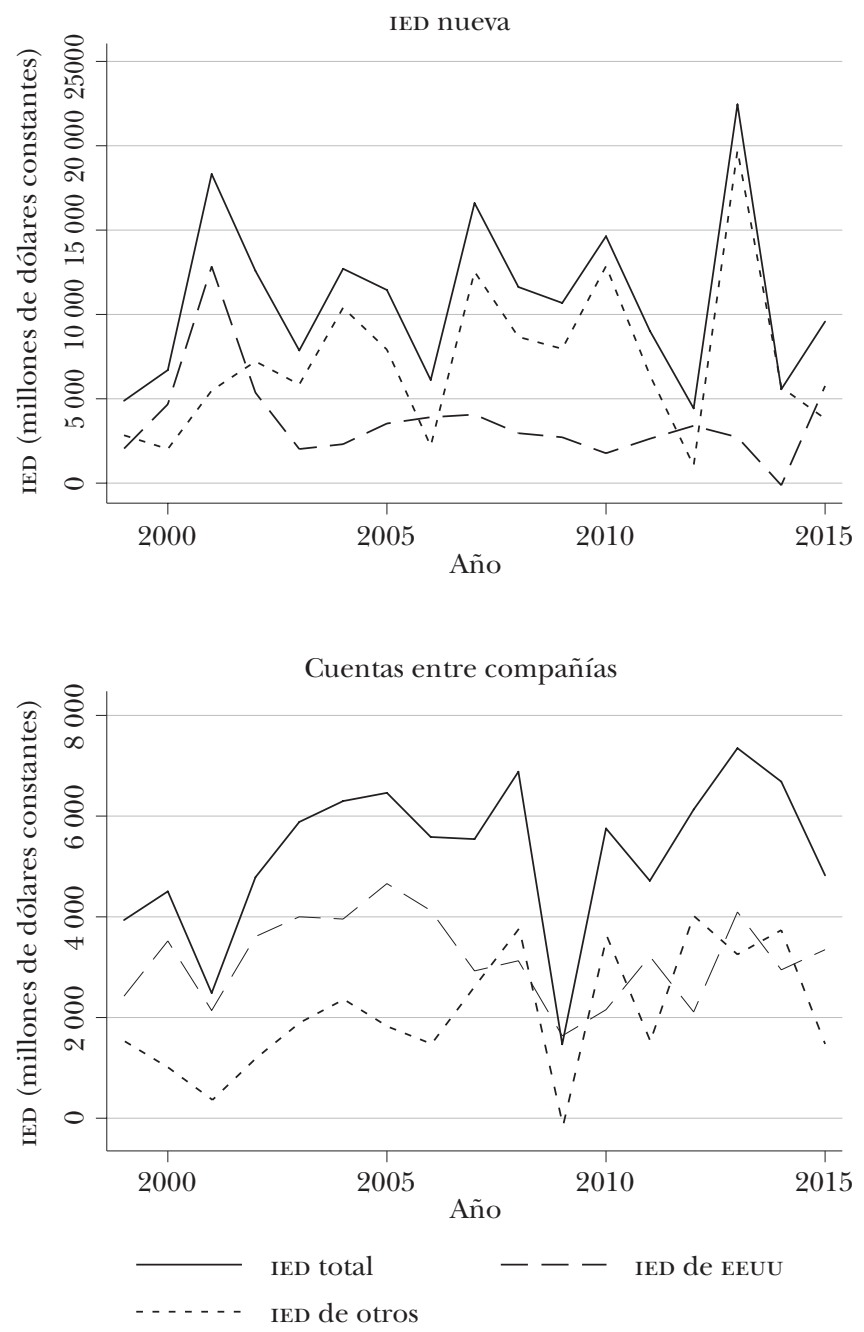

Fuente: Elaboración propia con datos de la Dirección General de Inversión Extranjera, de la Secretaría de Economía.

Nota: Las cifras correspondientes al año 2015 sólo reflejan los tres primeros trimestres del año. 


\section{GRÁFICA 3}

IED procedente de los EE.UU., como porcentaje de la IED total anual

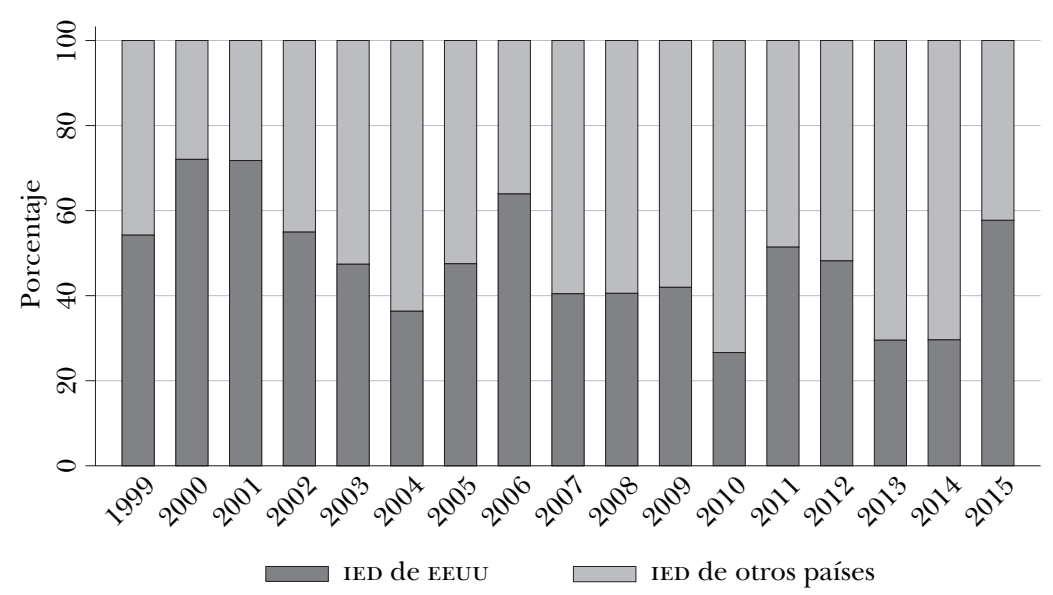

Fuente: Elaboración propia con datos de la Dirección General de Inversión Extranjera, de la Secretaría de Economía.

Nota: Las cifras correspondientes al año 2015 sólo reflejan los tres primeros trimestres del año.

\subsection{El mosaico subnacional}

Es necesario tener en cuenta que la participación de la IED estadounidense en los flujos de IED recibida no sólo ha tenido una variación interesante a lo largo del tiempo, sino que ha manifestado una heterogeneidad espacial notable. En esta sección se analizan las características de la IED procedente de los EE.UU. y del resto del mundo a nivel subnacional, para identificar diferencias en las dinámicas espaciales de la inversión. Si se analiza el periodo en su conjunto, utilizando dólares constantes de 2012, seis estados recibieron casi el $80 \%$ de la IED total. La Ciudad de México, Nuevo León, Estado de México, Jalisco, Chihuahua y Colima recibieron, respectivamente, el $49.9 \%, 8.8 \%, 4.9 \%, 4.9 \%, 4.5 \%$ y $4.1 \%$ de la IED de este periodo. ${ }^{36}$

${ }^{36}$ Nótese que el ordenamiento es distinto si se utilizan dólares corrientes o 
El mapa 1 ilustra la IED total recibida por los estados mexicanos, por quintiles. ${ }^{37}$

Sin embargo, hay algunas diferencias entre los estados que tienen la mayor cantidad de la IED de los EE.UU. y del resto del mundo. Los estados que tienen mayor IED de los EE.uU. son la Ciudad de México (54.7\%), Chihuahua (8.6\%), Nuevo León (7.4\%) y Baja California (7.3\%). El mapa 2 ilustra estas dinámicas. Es interesante destacar, en fin, que no se observan diferencias marcadas en la IED en los estados de frontera por origen. Esto sugeriría que las oportunidades de acceso al mercado de los EE.UU. abiertas por el TLCAN han sido aprovechadas no sólo (o no de manera desproporcionadamente mayor) por inversionistas de origen estadounidense. Sin embargo, el análisis multivariado que se presenta al final de esta sección sugiere que tales diferencias se dan después de controlar el efecto de otros determinantes de la IED.

Con ciertos contrastes, los estados que atraen el mayor volumen de IED de otro origen son el Distrito Federal (56.8\%), Nuevo León (11.5\%), Jalisco (6.2\%), Hidalgo (4.02\%), Puebla (2.5\%) y Querétaro (1.8\%). Sin embargo, los contrastes son aún mayores, si se compara la importancia relativa de cada estado en los flujos de IED de distinto origen. El cuadro 1 muestra la clasificación de los estados según el origen de la IED total recibida en el periodo bajo estudio. Los estados se ordenaron de mayor a menor, según la IED total recibida entre 1999 y 2013 en dólares de 2012. Una rápida comparación de las dos últimas columnas muestra que hay una varianza significativa en la localización de las inversiones procedentes de los EE.UU. y la de otro origen. Estas diferencias se registran aun entre los estados que reciben los mayores volúmenes de IED total y se hacen todavía más marcadas aún según se desciende en el cuadro.

distintos periodos. Sin embargo, el uso de dólares constantes proporciona un panorama más adecuado de la IED recibida en este periodo.

${ }^{37}$ Para elaborar estos mapas, se ordenaron los estados según el volumen total de IED recibida en este periodo, en dólares constantes de 2012, y se los subdividió en grupos de 6 ó 7 estados (quintiles) para facilitar la representación gráfica de los niveles de IED en los que cada estado se ubica. Para la elaboración de los mapas, se omitió el año 2013, ya que una sola operación (la compra del grupo Modelo) alteraba resultados que son consistentes a lo largo de todo el periodo examinado. 


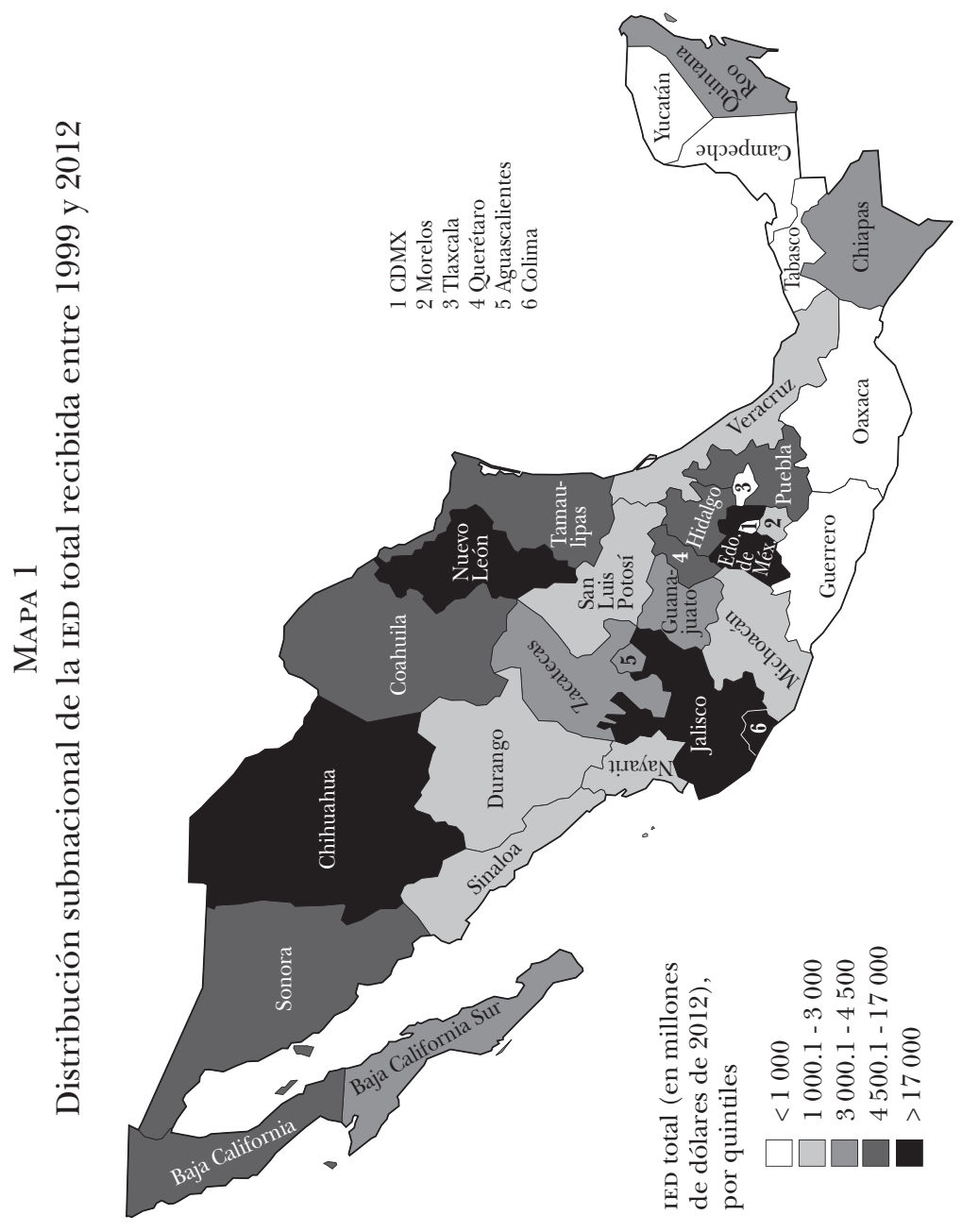




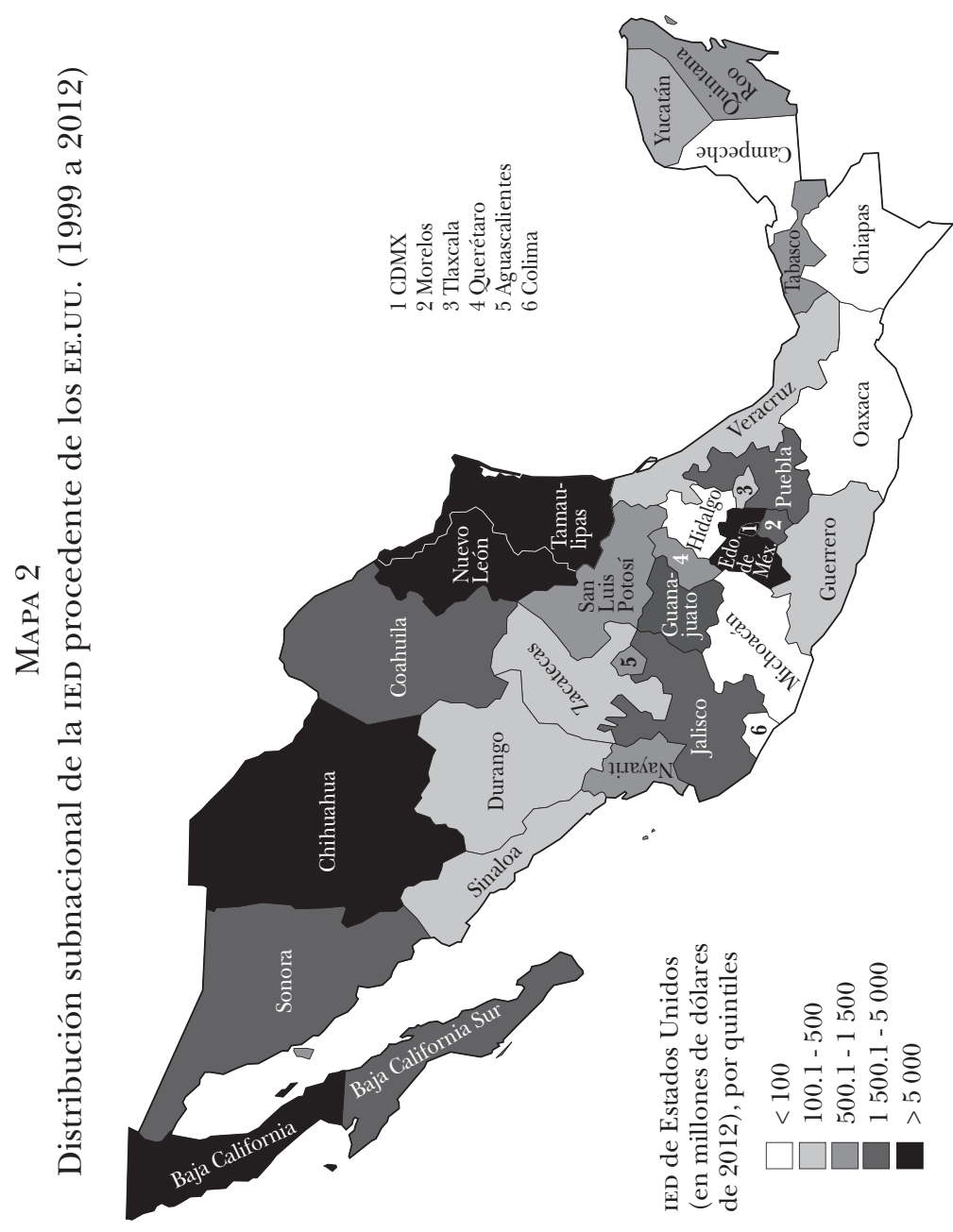




\section{Cuadro 1}

Clasificación de estados según la IED total recibida entre 1999 y 2013, en valores constantes

\begin{tabular}{|c|c|c|c|}
\hline & IED total & IED de los EE.UU. & IED del resto del mundo \\
\hline Distrito Federal & 1 & 1 & 1 \\
\hline Nuevo León & 2 & 3 & 2 \\
\hline Estado de México & 3 & 5 & 13 \\
\hline Jalisco & 4 & 7 & 3 \\
\hline Chihuahua & 5 & 2 & 23 \\
\hline Colima & 6 & 27 & 10 \\
\hline Baja California & 7 & 4 & 17 \\
\hline Hidalgo & 8 & 31 & 4 \\
\hline Puebla & 9 & 10 & 5 \\
\hline Tamaulipas & 10 & 6 & 20 \\
\hline Coahuila & 11 & 9 & 25 \\
\hline Sonora & 12 & 11 & 11 \\
\hline Querétaro & 13 & 14 & 6 \\
\hline Baja California Sur & 14 & 8 & 19 \\
\hline Guanajuato & 15 & 12 & 21 \\
\hline Chiapas & 16 & 30 & 15 \\
\hline Quintana Roo & 17 & 15 & 8 \\
\hline Aguascalientes & 18 & 18 & 9 \\
\hline Zacatecas & 19 & 24 & 7 \\
\hline San Luis Potosí & 20 & 16 & 16 \\
\hline Durango & 21 & 22 & 12 \\
\hline Michoacán & 22 & 29 & 14 \\
\hline Morelos & 23 & 13 & 32 \\
\hline Veracruz & 24 & 25 & 18 \\
\hline Nayarit & 25 & 17 & 24 \\
\hline Sinaloa & 26 & 21 & 22 \\
\hline Tabasco & 27 & 19 & 29 \\
\hline Yucatán & 28 & 20 & 30 \\
\hline Tlaxcala & 29 & 23 & 26 \\
\hline Oaxaca & 30 & 28 & 27 \\
\hline Guerrero & 31 & 26 & 28 \\
\hline Campeche & 32 & 32 & 31 \\
\hline
\end{tabular}

Nota: En negritas, se destacan las cinco primeras posiciones en la clasificación y, en cursivas, en celdas sombreadas, las cinco últimas. 
Un último análisis, el descriptivo, tiene que ver no tanto con qué estados son más atractivos para inversionistas de distinto origen, como con la presencia relativa de inversionistas de distinto origen en cada estado.

El mapa 3 permite visualizar la importancia de los flujos de IED de los EE.UU. como porcentaje de la IED total de cada entidad federativa. Cinco estados mexicanos deben más del $85 \%$ de los flujos totales de IED, que recibieron durante este periodo, a inversiones de los EE.uU. Ése es el caso de Morelos, cuya IED del periodo es casi por completo estadounidense, Baja California, Baja California Sur, Tamaulipas y Chihuahua. Evidentemente, estos estados son los más vulnerables a cualquier circunstancia (económica o política) que afecte los flujos de inversión en los EE.UU., aun cuando el volumen total de inversión de los mismos sea relativamente bajo (durante los 15 años examinados, estos cuatro estados recibieron casi el $22 \%$ de la inversión total procedente de los EE.UU., en tanto que sólo recibieron el $2.25 \%$ de la IED procedente del resto del mundo).

En el otro extremo se encuentran los estados en los que la IED estadounidense representa una mínima fracción de la IED total e incluso presentan saldos negativos de esta inversión en este periodo. Tal es el caso de Colima y Michoacán. Estas entidades, en conjunto, han recibido sólo el $0.07 \%$ del total de la IED de EE.UU. en México y el $2.3 \%$ de la IED de otro origen. Más extremos son los casos de Campeche, Chiapas e Hidalgo, cuyo balance, al cabo de estos 14 años, es negativo (han perdido IED procedente de los EE.UU. que ya existía en sus territorios, aun cuando esos flujos se compensaron con IED de otro origen).

\subsection{Tendencias nacionales}

La gráfica 4 muestra las líneas de tendencias de los datos presentados en la gráfica 2. Téngase presente que hay que tener cierta cautela al interpretar líneas de tendencia en muestras que sólo incluyen quince años. Sin embargo, estas trayectorias simplificadas ayudan a la visualización de datos descriptivos, en especial cuando la tendencia no es lineal. En los dos paneles superiores, se agrega 


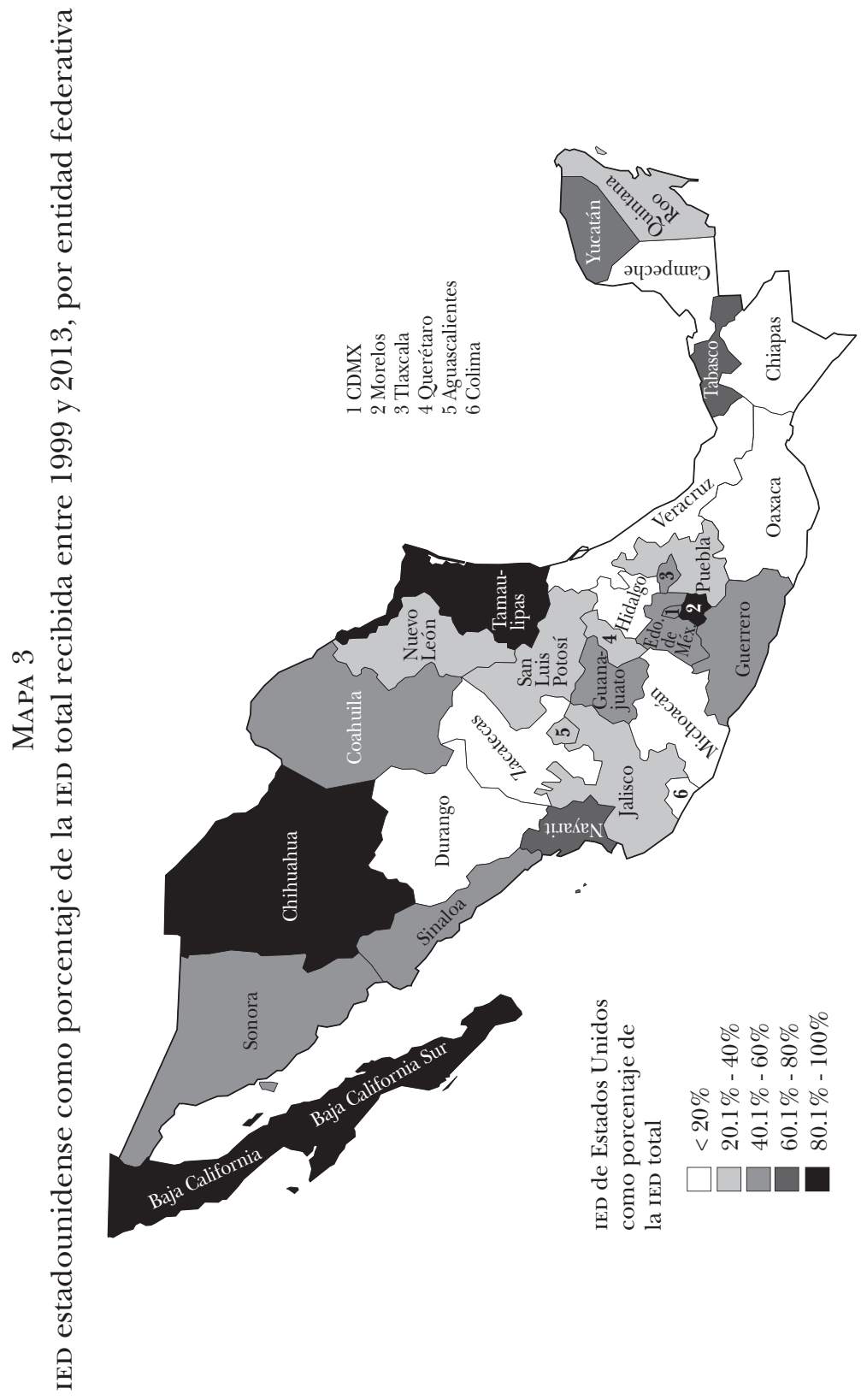




\section{Gráfica 4}

Tendencia de IED de EE.UU. y del resto del mundo (2000-2014)
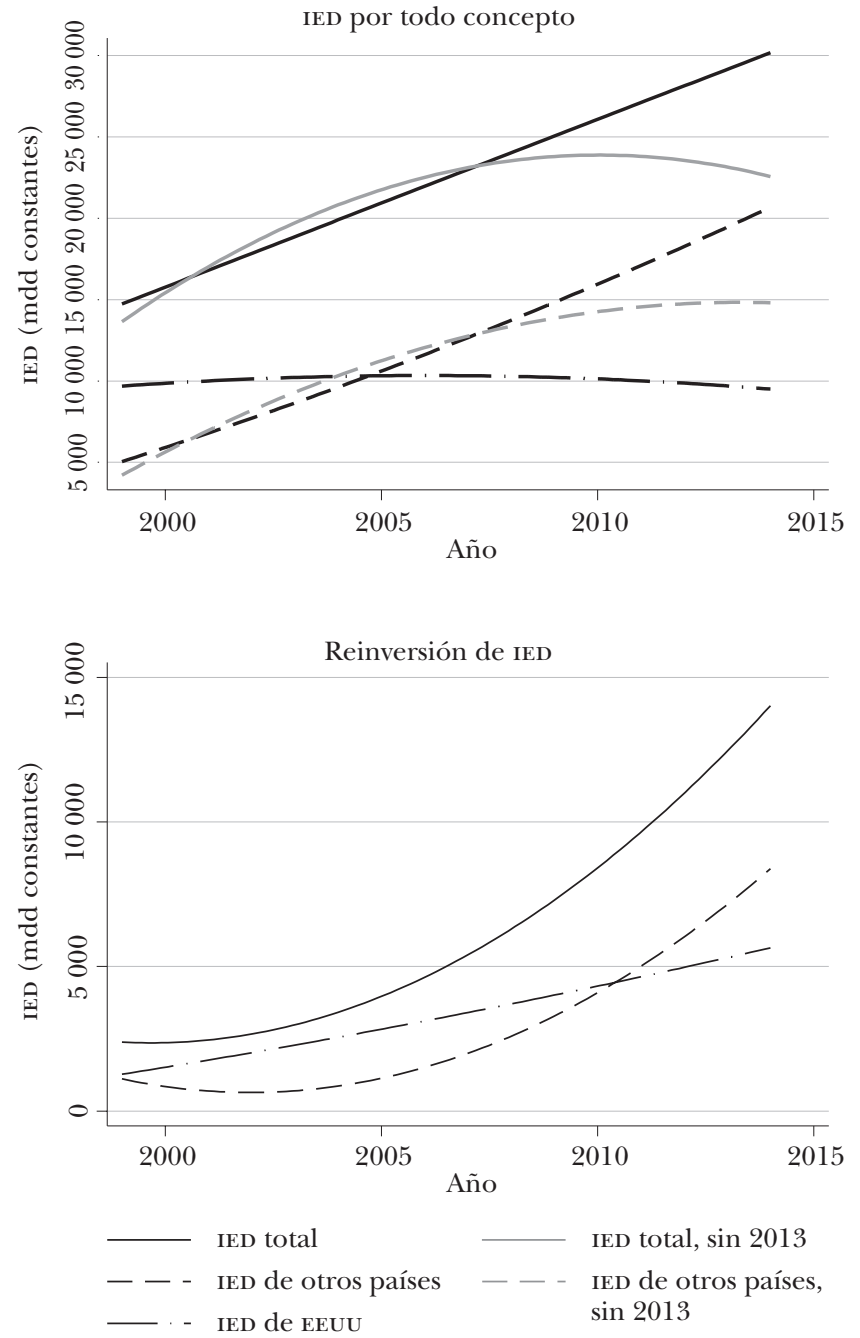

Fuente: Elaboración propia con datos de la Dirección General de Inversión Extranjera, de la Secretaría de Economía.

Nota: La línea de tendencia representa el promedio con ajuste cuadrático (quadratic fit). 
GRÁfICA 4 (concluye)

Tendencia de IED de EE.UU. y del resto del mundo (2000-2014)
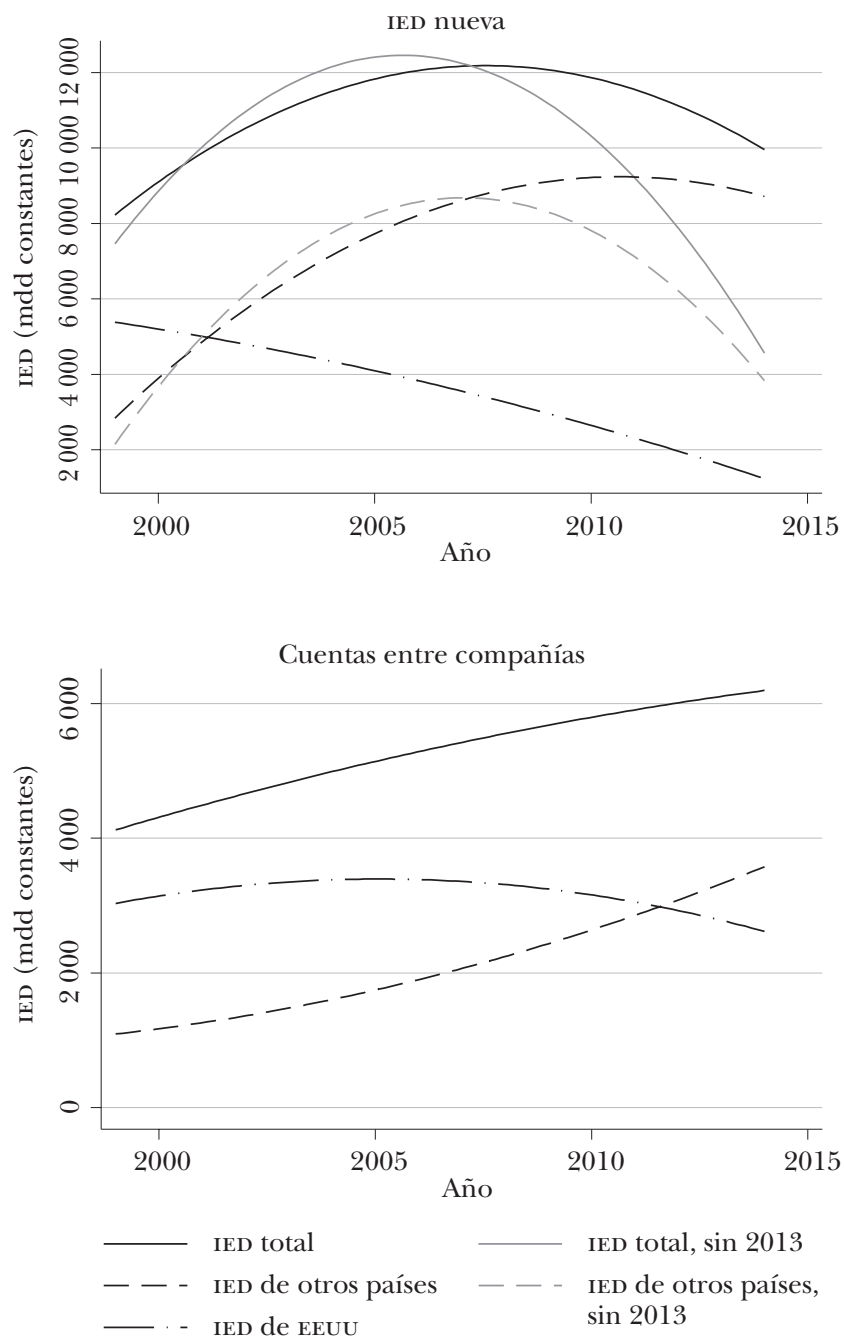

Fuente: Elaboración propia con datos de la Dirección General de Inversión Extranjera, de la Secretaría de Economía.

Nota: La línea de tendencia representa el promedio con ajuste cuadrático (quadratic fit). 
una cuarta línea que calcula la trayectoria de la IED del resto del mundo en la que se omite el año 2013 para los casos de inversión por todo concepto y nueva IED. Esto se debe a que la inusual magnitud de la compra de la cervecería Modelo claramente altera el cálculo de la tendencia de la IED global y procedente del resto del mundo. Entendemos que las trayectorias calculadas, al omitirse 2013, muestran un panorama más cercano a la realidad.

En el primer panel de esta gráfica (IED por todo concepto) puede observarse que la tendencia de los flujos totales de IED en México parece ser ascendente. Sin embargo, esto es producto, en gran medida, del año 2013. La segunda línea (IED total sin 2013) muestra que el flujo total de inversiones se ha mantenido estable en término reales desde el año 2009, con una tendencia a la baja hacia el final del periodo. La tendencia general de la IED procedente de los EE.UU. es relativamente estable, manteniéndose en promedio alrededor de los 10,000 mdd anuales. ${ }^{38}$ La IED procedente del resto del mundo tenía una trayectoria ascendente hasta que se desató la crisis a finales de 2007. Si se omiten las cifras de 2013 del cálculo, la gráfica sugiere que los flujos procedentes del resto del mundo han tendido a estabilizarse a partir del año 2009. Esta observación no implica argumentar que la crisis financiera mundial sea la única causa del cambio en la tendencia al alza de la IED dirigida a México, ya que otros factores domésticos, tales como el incremento en la violencia en algunos estados, ciertamente han podido contribuir con esta tendencia. ${ }^{39}$

Es interesante mirar en conjunto los tres paneles restantes de la gráfica 4. Como puede apreciarse, si bien la IED nueva procedente de los EE.UU. ha caído drásticamente en el periodo analizado, esta caída se ve compensada por el crecimiento sostenido de la reinversión de fondos procedentes de los EE.UU. Las cuentas entre compañías estadounidenses también registran una baja hacia el final del periodo, pero la magnitud es pequeña en comparación con los otros flujos.

${ }^{38}$ Cabe destacar que la tendencia que se presenta para la IED de los EE.UU. no cambia fundamentalmente, si se calcula eliminando la observación extrema correspondiente al año 2001.

${ }^{39}$ Escobar Gamboa, art. cit. 
Las inversiones procedentes del resto del mundo se comportan de manera diferente según los componentes de los flujos totales. La trayectoria de la IED total procedente del resto del mundo es principalmente una consecuencia de nuevas inversiones, apoyada también por decisiones de reinversión y de cuentas entre compañías. La tendencia al alza de las nuevas inversiones se revierte en 2012 (en 2007, si omitimos los valores de 2013), pero esto se compensa con los otros dos flujos.

\subsection{Tendencias subnacionales}

No es posible reproducir aquí, por el espacio de que se dispone, el análisis anterior de cada unidad subnacional. Sin embargo, es necesario destacar que las dinámicas subnacionales generalmente no replican el agregado nacional. Por ejemplo, aun cuando los flujos totales de IED desde los EE.UU. hacia México se han mantenido a la baja relativamente constantes en el periodo estudiado, si se comparan los flujos de IED estadounidenses recibidos al inicio y al final del periodo examinado, quince estados aumentaron considerablemente en términos reales la inversión recibida del vecino del norte. El mapa 4 ilustra cuáles estados ganaron y cuáles perdieron IED de los EE.UU. en este periodo, así como la magnitud del cambio en los flujos recibidos. ${ }^{40}$

Como puede apreciarse, veintitrés entidades federativas han registrado caídas en la IED que recibieron de los EE.UU. En particular, cuatro estados han recibido menos del $75 \%$ de la IED que recibían de los EE.uU. a principios del periodo analizado. Como en los casos de Hidalgo y Campeche (que registraron flujos negativos de IED estadounidense durante 2011-2013), Sonora y Yucatán. Por el contrario, de los quince estados que tuvieron incrementos reales en los flujos de IED procedentes de los EE.UU., Tlaxcala, Baja California

${ }^{40}$ Para evitar distorsiones provocadas por operaciones de excepcional magnitud en el primer o último año del periodo considerado, se compararon los promedios de los tres primeros y de los tres últimos años del periodo (1999 a 2001 y 2001 a 2013). 


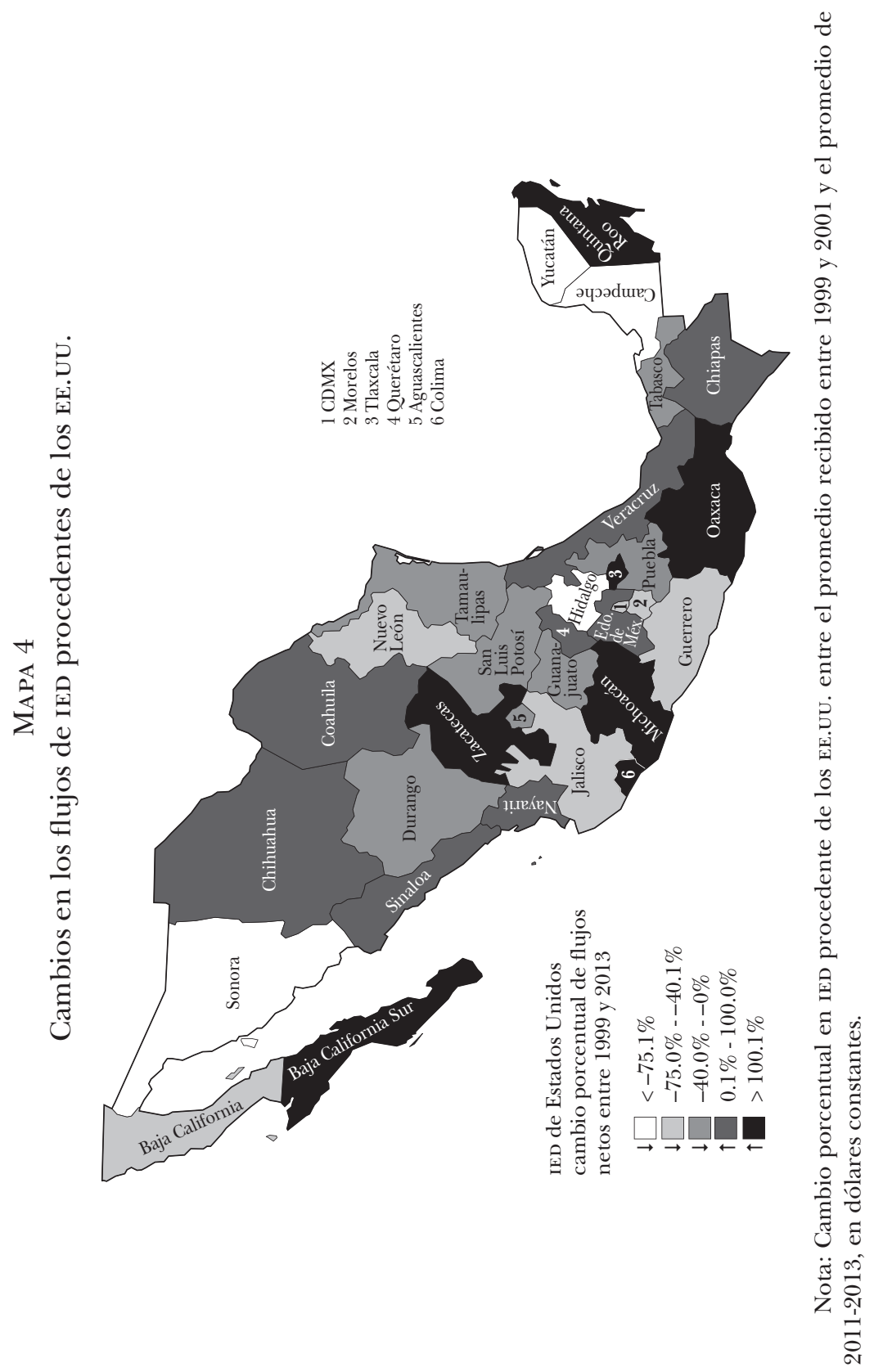


Sur, Oaxaca, Colima y Quintana Roo duplicaron o triplicaron esos flujos, en tanto que Zacatecas y Michoacán multiplicaron las inversiones anuales promedio recibidas al inicio del periodo en términos reales por 3 y 17 , respectivamente.

\section{5. ¿Distintos determinantes?}

Una última cuestión por dilucidar es qué factores podrían explicar estas diferencias de comportamiento entre la IED procedente de los EE.uU. y la del resto del mundo. La elaboración de una teoría sobre los factores que determinan la inversión extranjera de distinto origen escapa al objeto de este trabajo. Sin embargo, es posible examinar si las correlaciones entre factores que normalmente se asocian con la atracción de IED muestran diferencias según el origen de los flujos.

El cuadro 2 presenta los resultados de una serie de regresiones que explora el efecto de determinantes de la localización subnacional de la IED, según su origen. La variable dependiente es IED recibida por el estado como proporción de su producto interno bruto (PIB). Los modelos 1 y 3 explican la IED procedente de los EE.UU., y los modelos 2 y 4 , la IED procedente del resto del mundo. Los modelos incluyen controles comunes en la bibliografía citada, tales como la variable dependiente retrasada un año, el tamaño del mercado (medido como población estatal), el nivel de desarrollo (Рів per capita) y el crecimiento del PIв. Se incluye una medida de capital humano (años promedio de escolarización) y dos medidas de capital físico (la existencia de puertos comerciales y la densidad de carreteras). Dos variables dicotómicas indican si el estado en consideración es la Ciudad de México o se encuentra en la frontera con los EE.UU. La variable Tendencia es un contador del número de años transcurridos desde el inicio de la muestra. Los datos se obtuvieron del INEGI.

Los modelos 3 y 4 incorporan variables políticas. La variable Alternancia indica si el Partido Revolucionario Institucional (PRI) perdió el control de la gubernatura en algún momento. La "regla 
Cuadro 2

Determinantes de la IED de distinto origen. Estados mexicanos, 1999-2013

\begin{tabular}{|c|c|c|c|c|}
\hline & $\begin{array}{c}\text { Modelo } 1 \\
\text { EE.UU. }\end{array}$ & $\begin{array}{c}\text { Modelo } 2 \\
\text { Resto del mundo }\end{array}$ & $\begin{array}{c}\text { Modelo } 3 \\
\text { EE.UU. }\end{array}$ & $\begin{array}{c}\text { Modelo } 4 \\
\text { Resto del mundo }\end{array}$ \\
\hline Variable dependente $_{\mathrm{t}-1}$ & $\begin{array}{c}.256 \\
(5.52) * * *\end{array}$ & $\begin{array}{c}.141 \\
(2.89)^{* * * *}\end{array}$ & $\begin{array}{c}.271 \\
(5.88) * * *\end{array}$ & $\begin{array}{c}.120 \\
(2.47) * *\end{array}$ \\
\hline Tamaño del mercado $\mathrm{t}-1$ & $\begin{array}{c}-.237 \\
(-1.75)^{*}\end{array}$ & $\begin{array}{l}-.152 \\
(-1.11)\end{array}$ & $\begin{array}{l}-.170 \\
(-1.29)\end{array}$ & $\begin{array}{l}-.130 \\
(-.92)\end{array}$ \\
\hline Desarrollo $_{\mathrm{t}-1}$ & $\begin{array}{c}-.006 \\
(-.62) * * *\end{array}$ & $\begin{array}{l}-.007 \\
(-.64)\end{array}$ & $\begin{array}{l}-.003 \\
(-.29)\end{array}$ & $\begin{array}{l}-.007 \\
(-.57)\end{array}$ \\
\hline Crecimiento $_{\mathrm{t}-1}$ & $\begin{array}{c}.00009 \\
(.06)\end{array}$ & $\begin{array}{l}-.0003 \\
(-.12)\end{array}$ & $\begin{array}{l}.0002 \\
(.10)\end{array}$ & $\begin{array}{l}-.0008 \\
(-.34)\end{array}$ \\
\hline Escolarización $_{\mathrm{t}-1}$ & $\begin{array}{c}.152 \\
(1.33)\end{array}$ & $\begin{array}{c}.177 \\
(1.33)\end{array}$ & $\begin{array}{c}.141 \\
(1.26)\end{array}$ & $\begin{array}{c}.192 \\
(1.43)\end{array}$ \\
\hline Puertos & $\begin{array}{l}-.040 \\
(-.20)\end{array}$ & $\begin{array}{c}-.525 \\
(-2.59) * *\end{array}$ & $\begin{array}{l}.174 \\
(.82)\end{array}$ & $\begin{array}{c}-.459 \\
(-2.00)^{* *}\end{array}$ \\
\hline Densidad de carreteras & $\begin{array}{c}-.75 \\
(-1.25)\end{array}$ & $\begin{array}{l}-1.225 \\
(-1.91)^{*}\end{array}$ & $\begin{array}{l}-.376 \\
(-.63)\end{array}$ & $\begin{array}{l}-1.089 \\
(-1.60)\end{array}$ \\
\hline Frontera & $\begin{array}{c}.784 \\
(2.59)^{* *}\end{array}$ & $\begin{array}{l}-.222 \\
(-.71)\end{array}$ & $\begin{array}{c}.852 \\
(2.89) * * *\end{array}$ & $\begin{array}{l}-.204 \\
(-.64)\end{array}$ \\
\hline CDMX & $\begin{array}{c}2.661 \\
(3.96)^{* * * *}\end{array}$ & $\begin{array}{c}2.790 \\
(4.01)^{* * *}\end{array}$ & $\begin{array}{c}2.443 \\
(3.77)^{* * * *}\end{array}$ & $\begin{array}{c}2.769 \\
(3.94)^{* * *}\end{array}$ \\
\hline Alternancia $_{\mathrm{t}-1}$ & & & $\begin{array}{c}.432 \\
(2.45)^{* *}\end{array}$ & $\begin{array}{l}.147 \\
(.71)\end{array}$ \\
\hline Calderón & & & $\begin{array}{c}-.209 \\
(-1.02)\end{array}$ & $\begin{array}{c}.764 \\
(2.61)^{* * *}\end{array}$ \\
\hline Peña Nieto & & & $\begin{array}{l}-.015 \\
(-.05)\end{array}$ & $\begin{array}{c}.992 \\
(2.17)^{* *}\end{array}$ \\
\hline Tendencia & $\begin{array}{c}-.045 \\
(-2.17) * *\end{array}$ & $\begin{array}{l}.025 \\
(.99)\end{array}$ & $\begin{array}{l}-.042 \\
(-1.30)\end{array}$ & $\begin{array}{c}-.068 \\
(-1.56)\end{array}$ \\
\hline Constante & $\begin{array}{l}3.313 \\
(1.43)\end{array}$ & $\begin{array}{l}1.921 \\
(.79)\end{array}$ & $\begin{array}{c}2.035 \\
(.90)\end{array}$ & $\begin{array}{l}1.656 \\
(.66)\end{array}$ \\
\hline Observaciones & 448 & 448 & 448 & 448 \\
\hline $\mathrm{R}^{2}$ intra-grupos & .133 & .037 & .145 & .048 \\
\hline $\mathrm{R}^{2}$ entra-grupos & .760 & .723 & .792 & .720 \\
\hline $\mathrm{R}^{2}$ global & .528 & .271 & .552 & .276 \\
\hline
\end{tabular}

Nota: Regresión de mínimos cuadrados generalizados para paneles, con corrección para distorsiones autorregresivas (AR1). Valores $Z$ entre paréntesis. La significancia estadística se indica como: $* \mathrm{p}<.1, * * \mathrm{p}<.05, * * * \mathrm{p}<.01$. 
de alternancia" en el poder ${ }^{41}$ se usa como una indicación, si bien imperfecta, de democracia a nivel subnacional. Se incorporan, en fin, dos variables dicotómicas (Calderón y Peña Nieto) para comparar la IED recibida bajo estas administraciones, en comparación con la de la administración de Fox. La estimación es una regresión de mínimos cuadrados generalizados para paneles, con corrección para distorsiones autorregresivas (AR1).

La falta de significación estadística de muchos de los controles no debe sorprender por dos motivos: la inclusión de la variable dependiente retrasada un año absorbe mucho del efecto atribuible a características relativamente constantes de los estados; segundo, la mayoría de los estudios sobre distribución subnacional de la IED no encuentran resultados robustos para estas variables.

$\mathrm{El}$ análisis muestra interesantes diferencias entre los factores que la IED procedente de distinto origen. En primer lugar, la IED procedente de los EE.uU. depende más de decisiones pasadas sobre inversión que la del resto del mundo. El coeficiente asociado a la variable retrasada para IED de los EE.UU. dobla en magnitud el de los modelos 2 y 4 . Esto puede entenderse como consecuencia de la dependencia temporal de las decisiones de inversión, pero el efecto más acentuado en el caso de la IED procedente de EE.UU. sugiere la presencia de un efecto informacional entre inversionistas de la misma nacionalidad que probablemente no se repita cuando el grupo sea más heterogéneo. ${ }^{42}$

En relación con lo que toca a la infraestructura física, los modelos sugieren que las variables incluidas no afectan la inversión estadounidense, pero sí la procedente del resto del mundo. La dirección del efecto es el contrario al que se esperaría (los inversionistas del resto del mundo evitan los estados con puertos comerciales y con

${ }^{41}$ Adam Przeworski et al., Democracy and Development. Political Institutions and Well-Being in the World, 1950-1990, Cambridge, MA, Cambridge University Press, 2000.

42 A. C. Garriga y B. J. Phillips, "Foreign Aid as a Signal to Investors: Predicting FDI in Post-conflict Countries", Journal of Conflict Resolution, vol. 58, núm. 2, 2014, pp. 280-306, y Ashoka Mody, Efraim Sadka y Assaf Razin, The Role of Information in Driving FDI Flows: Host-Country Transparency and Source-Country Specialization, Washington, DC, International Monetary Fund, 2003. 
mayor densidad carretera ${ }^{43}$ ), pero también es consistente con la bibliografía especializada previa.

A pesar de que la evidencia cualitativa no señalaba diferencias por origen en la preferencia por los estados que limitan con los EE.UU., el análisis multivariado sugiere que, al mantenerse otros factores constantes, la inversión estadounidense se dirige en mayor medida a los estados fronterizos. La frontera no tiene un efecto significativo sobre la IED del resto del mundo. Por lo demás, el Modelo 1 muestra que la tendencia de la IED de los EE.UU. es negativa, aunque este efecto no es significativo para la inclusión de otras variables que también tienen un efecto temporal.

Las diferencias más marcadas entre los determinantes de estos dos grupos de inversionistas aparecen cuando se incluyen las variables políticas. Los inversionistas estadounidenses prefieren estados que han experimentado alternancia partidaria, el (rudimentario) indicador de democracia subnacional. Sin embargo, la alternancia no afecta las inversiones del resto del mundo. En segundo lugar, los flujos de inversión estadounidense no se vieron alterados por cambios en el gobierno federal. Sin embargo, la IED procedente del resto del mundo aumentó en las administraciones de Calderón y de Peña Nieto (comparadas con la administración de Fox). Toda vez que este efecto es significativo cuando se controla por tendencia, se interpreta que es independiente al mero paso del tiempo. Sin embargo, ya que el único año incluido en la muestra bajo el Presidente Peña Nieto es 2013, es posible que el efecto que observamos se deba al singular efecto de la compra del grupo Modelo en ese año.

\section{Conclusiones}

Este trabajo se ha propuesto distinguir las características de la IED recibida por México de los EE.Uu. y la del resto del mundo. El análisis descriptivo y estadístico permite afirmar que hay indicios

\footnotetext{
${ }^{43}$ Este último resultado, sin embargo, no es robusto.
} 
de un comportamiento diferencial en la IED de origen estadounidense y en la de otro origen.

Una primera conclusión es que los patrones temporales de IED difieren en función de su origen. La IED procedente de los EE.UU. en términos reales es relativamente estable, al mantenerse en promedio alrededor de los 10,000 mdd anuales (dólares de 2012), lo que contrasta con el rápido crecimiento de las inversiones de otro origen hasta fines de 2007, cuando estos flujos muestran los efectos de la crisis financiera mundial a partir de 2008. Si se omite el año de 2001, el cual constituye una marca para la IED estadounidense, puede observarse que las inversiones procedentes del resto del mundo, aun cuando son más diversificadas, implican flujos de capital más volátiles y cíclicos que la IED procedente de los EE.uU. Una parte interesante del presente estudio es el análisis de las causas de esa estabilidad a pesar de los vaivenes de la economía estadounidense en el periodo examinado. Es posible que el horizonte temporal de las inversiones de distinto origen sea desigual o que inversionistas de otro origen se concentren en actividades económicas particulares que requieran, por ejemplo, reinversiones sostenidas.

También se advierten diferencias según su origen si se divide la IED por tipos de flujo (inversión nueva, reinversiones y cuentas entre compañías). El deterioro de las nuevas inversiones de ambos orígenes es un dato preocupante. Si bien México sigue siendo un destino importante para la IED, esta tendencia parece ser el resultado, en gran medida, de decisiones de reinversión y no de la atracción de nuevos capitales. Este dato tiene varias implicancias de política pública. En primer lugar, es necesario diferenciar las políticas de atracción de nuevas inversiones de las políticas de arraigamiento o reinversión. Las diferencias de IED nueva según su origen conllevan analizar las causas de la caída en las IED estadounidenses. Es posible que se trate de un fenómeno de "regresión a la media" tras el crecimiento de IED y la firma del TLCAN, pero es posible que también otros factores estén afectando las decisiones de inversionistas del norte. Asimismo, el fenómeno de compensación entre IED nueva y reinversión sugiere que la política de atracción de inversiones se beneficiaría con la inclusión 
de garantías que estimulen la reinversión y que permitan sostener la tendencia al alza que ya es evidente.

Otro dato interesante es que, aunque la tendencia general sea la disminución de la IED estadounidense, hay estados que han podido mantener, e incluso incrementar, estos flujos de inversión. El análisis de esos casos puede ilustrar estrategias de atracción más exitosas, así como el desarrollo de actividades industriales y de servicios que son más atractivas para los inversionistas estadounidenses. Los datos presentados aquí también son indicadores de la distinta vulnerabilidad de los estados mexicanos a las circunstancias económicas de los EE.uU. La estimación del impacto de la IED estadounidense en términos relativos es un dato que se debe tener especialmente en cuenta por sobre el de aquellos estados en que la IED estadounidense es más alta en términos reales. En particular, hay cinco estados (identificados en el Mapa 3) cuya fuente casi exclusiva de IED son los EE.uU., lo que los hace extremadamente vulnerables a circunstancias exógenas. Quizás sería conveniente que los gobiernos locales intentaran estrategias de diversificación de la IED para suavizar eventuales ciclos en la economía de los EE.UU. En este sentido, algunos estudios sugieren el éxito de estrategias de internacionalización de los entes subnacionales mediante actividades de paradiplomacia. Este instrumento permitiría a gobiernos locales dirigir la búsqueda de inversiones a países específicos. ${ }^{44}$

Si bien este artículo no ha pretendido estudiar las determinantes de los distintos flujos de inversión, el análisis multivariado sugiere que los factores que atraen la IED de distinto origen no son idénticos, lo que investigaciones futuras deberán cuestionarse cuando analicen las causas de estas diferencias y, en particular, el llamativo hallazgo del efecto diferencial de la alternancia partidaria

${ }^{44}$ Ivo Duchacek, "The International Dimension of Subnational Self-Government”, Publius, vol. 38, núm. 14, 1984, pp. 5-31; Brian Hocking, "Patroling the «Frontier»: Globalization, Localization, and the «Actorness» of Non-Central Governments", en Francisco Aldecoa y Michael Keating (eds.), Paradiplomacy in Action: The Foreign Relations of Subnational Governments, Londres, Frank Cass, 1999, pp. 17-38; Jorge Schiavon, La Proyección Internacional de las Entidades Federativas: México ante el Mundo, México, Secretaría de Relaciones Exteriores, 2006. 
subnacional. El presente estudio, además, tiene una serie de limitaciones por la necesidad de acotar la pregunta de investigación ${ }^{45}$. Su propósito ha sido descriptivo, por lo cual no puede sustentar juicios de causalidad. Sin embargo, contar con descripciones detalladas, enmarcadas teóricamente, es un primer paso para la elaboración de teorías que expliquen las diferencias encontradas. Asimismo, este tipo de descripciones constituye en sí mismo un insumo para el diseño y aplicación de políticas públicas.

Otra de las limitaciones derivadas de la pregunta de investigación es concentrarse en diferencias entre la IED de los EE.UU. y la del resto del mundo. Evidentemente, es posible que inversionistas de otros países tengan también un comportamiento singular (ahora asimilado en la categoría "resto del mundo"). Sin embargo, la magnitud de las inversiones de los EE.UU. y una bibliografía especializada, que sugiere diferencias de la IED estadounidense, justifican esta primera aproximación a diferencias por origen. El estudio individualizado de otros orígenes de IED permitirá, sin duda, la elaboración de políticas de atracción y diversificación de inversiones mejor encaminadas.

Entendemos que este análisis presenta elementos para efectuar un diagnóstico más completo del fenómeno de la IED en México que revele la toma de decisiones en materia de atracción y retención de inversiones no sólo federales, sino también subnacionales. Esta información es oportuna, ya que el gobierno está ideando estrategias para la recuperación de los niveles IED, tal como lo muestra la prensa ${ }^{46}$, y contribuye a llenar un vacío en la bibliografía que estudia los flujos de IED a México. En el plano teórico, la evidencia aquí presentada sugiere la utilidad de estudiar tanto las causas como las consecuencias de los flujos de IED de distinto origen, lo cual ayudará a mejorar el diseño de políticas diferenciadas de atracción de inversiones y, con el tiempo, el de tipos de inversiones.

${ }^{45}$ Las limitaciones derivadas de los datos utilizados se discutieron en la sección 3.1. "El panorama nacional".

${ }^{46}$ Cf. http:/ / www.eluniversal.com.mx/finanzas/100907.html 


\section{BibLLIOGRAFÍA}

Aitken, Brian, Gordon H. Hanson y Ann E. Harrison, "Spillovers, Foreign Investment, and Export Behavior", Journal of International Economics 43, núms. 1-2, 1997, pp. 103-132.

Ando, Mitsuyo, Sven W. Arndt y Fukunari Kimura, "Production Networks in East Asia: Strategic Behavior by Japanese and US firms", manuscrito en ssRn, http://ssrn.com/abstract=981769 or http://dx.doi. org/10.2139/ssrn.981769

Aroca, Patricio y William F. Maloney, "Migration, Trade, and Foreign Direct Investment in Mexico", The World Bank Economic Review, vol. 19, núm. 3, 2005, pp. 449-472.

Biglaiser, Glen y Karl R. DeRouen, "Economic Reforms and Inflows of Foreign Direct Investment in Latin America", Latin American Research Review, vol. 41, núm. 1, 2006, pp. 51-75.

y Karl R. DeRouen, "Following the Flag: Troop Deployment and U.S. Foreign Direct Investment”, International Studies Quarterly, vol. 51, núm. 4, 2007, pp. 835-854.

y Joseph L. Staats, "Do Political Institutions Affect Foreign Direct Investment? A Survey of U.S. Corporations in Latin America”, Political Research Quarterly vol. 63, núm. 3, 2010, pp. 508-522.

— y David Lektzian, "The Effect of Sanctions on U.S. Foreign Direct Investment”, International Organization, vol. 65, núm. 3, 2011, pp. 531-551.

Borensztein, Eduardo, José de Gregorio y Jong-Wha Lee, "How Does Foreign Direct Investment Affect Economic Growth?”, Journal of International Economics, vol. 45, núm. 1, 1998, pp. 115-135.

Büthe, Tim y Helen V. Milner, "The Politics of Foreign Direct Investment into Developing Countries: Increasing FDI through International Trade Agreements?”, American Journal of Political Science, vol. 52, núm. 4, 2008, pp. 741-762.

Chidlow, Agnieszka, Laura Salciuviene y Stephen Young, "Regional Determinants of Inward FDI Distribution in Poland", International Business Review, vol. 18, núm. 2, 2009, pp. 119-133.

Cruz Gallegos, José Luis de la y José Antonio Núñez-Mora, "Comercio internacional, crecimiento económico e inversión extranjera directa: 
evidencias de causalidad para México", Revista de Economía Mundial, núm. 15, 2006, pp. 181-202.

, Carlos Canfield Rivera y Priscilla González Castro, "Economic Growth, Foreign Direct Investment And International Trade: Evidence on Causality in the Mexican Economy", Revista Brasileira de Economia de Empresas, vol. 9, núm. 1, 2009, pp. 17-29.

Cuevas, Alfredo, Miguel Messmacher y Alejandro Werner, "Foreign Direct Investment in Mexico since the Approval of nafta", The World Bank Economic Review, vol. 19, núm. 3, 2005, pp. 473-488.

Driffield, Nigel y James H Love, "Linking FDI motivation and host economy productivity effects: Conceptual and empirical analysis”, Journal of International Business Studies, vol. 38, núm. 3, 2007, pp. 460-473.

Duchacek, Ivo, "The International Dimension of Subnational Self-Government”, Publius, vol. 38, núm. 14, 1984, pp. 5-31.

Dunning, John H., "Toward an Eclectic Theory of International Production: Some Empirical Tests", Journal of International Business Studies, vol. 11, núm. 1, 1980, pp. 9-31.

, "The Eclectic Paradigm of International Production: A Restatement and Some Possible Extensions", Journal of International Business Studies, vol. 19, núm. 1, 1988, pp. 1-31.

Escobar Gamboa, Octavio Romano, "Foreign Direct Investment (FDI) Determinants and Spatial Spillovers across Mexico's States", The Journal of International Trade E Economic Development, vol. 22, núm. 7, 2013, pp. 993-1012.

Garriga, Ana Carolina, "Política partidaria e inversión extranjera directa. El Caso de los estados mexicanos", documento de trabajo DTEP, núm. 256, México, Centro de Investigación y Docencia Económicas, 2013.

y Brian J. Phillips, "Foreign Aid as a Signal to Investors: Predicting FDI in Post-conflict Countries", Journal of Conflict Resolution, vol. 58, núm. 2, 2014, pp. 280-306.

y Brian J. Phillips, "Criminal Violence and the Subnational Distribution of Foreign Direct Investment", documento de trabajo DTEP, núm. 279, México, Centro de Investigación y Docencia Económicas, 2015.

Globerman, Steven y Daniel M. Shapiro, "The Impact of Government Policies on Foreign Direct Investment: The Canadian Experience”, Journal of International Business Studies, vol. 30, núm. 3, 1999, pp. 513-532. 
Gutiérrez-Haces, Teresa, "La inversión extranjera directa en el TLCAN", Economía UNAM, vol.1, núm. 3, pp. 30-52.

Hocking, Brian, "Patroling the «Frontier»: Globalization, Localization, and the «Actorness» of Non-Central Governments", en Francisco Aldecoa y Michael Keating (eds.), Paradiplomacy in Action: The Foreign Relations of Subnational Governments, Londres, Frank Cass, 1999, pp. 17-38.

Javorcik, Beata S., Çă̆lar Özden, Mariana Spatareanu y Cristina Neagu, "Migrant Networks and Foreign Direct Investment", Journal of Development Economics, vol. 94, núm. 2, 2011, pp. 231-241.

Jensen, Nathan M., Nation-States and the Multinational Corporation. A Political Economy of Foreign Direct Investment, Princeton, NJ, Princeton University Press, 2006.

— y Guillermo Rosas, "Foreign Direct Investment and Income Inequality in Mexico, 1990-2000”, International Organization, vol. 61, núm. 3, 2007, pp. 467-487.

Jordaan, Jacob A. "Determinants of FDI-Induced Externalities: New Empirical Evidence for Mexican Manufacturing Industries”, World Development, vol. 33, núm. 12, 2005, pp. 2103-2118.

—, "Intra- and Inter-industry Externalities from Foreign Direct Investment in the Mexican Manufacturing Sector: New Evidence from Mexican Regions", World Development, vol. 36, núm. 12, 2008, pp. 28382854.

_ rect Investment: Evidence from Regional FDi in Mexico 1989-2006”, Growth and Change, vol. 39, núm. 3, 2008, pp. 389-413.

Kim, Chong-Sup, "Los efectos de la apertura comercial y de la inversión extranjera directa en la productividad del sector manufacturero mexicano”, El Trimestre Económico, vol. 64, núm. 255, 1997, pp. 365390.

Leblang, David, "Familiarity Breeds Investment: Diaspora Networks and International Investment”, American Political Science Review, vol. 104, núm. 3, 2010, pp. 584-600.

Li, Quan, "Democracy, Autocracy, and Tax Incentives to Foreign Direct Investors: A Cross-National Analysis", The Journal of Politics, vol. 68, núm. 1, 2006, pp. 62-74. 
Li, Xiaoying y Xiaming Liu, "Foreign Direct Investment and Economic Growth: An Increasingly Endogenous Relationship", World Development, vol 33, núm. 3, 2005, pp. 393-407.

Malesky, Edmund J., "Straight Ahead on Red: How Foreign Direct Investment Empowers Subnational Leaders", The Journal of Politics, vol. 70, núm. 1, 2008, pp. 97-119.

Márquez Pozos, Jorge Miguel, Alejandro Islas Camargo y Francisco Venegas-Martínez. "Corrientes internacionales de capital e inversión extranjera de cartera. El caso de México, 1989-1999”, El Trimestre Económico, vol. 70, núm. 280, 2003, pp. 791-833.

Meyer, Klaus E. y Hung Vo Nguyen, "Foreign Investment Strategies and Sub-national Institutions in Emerging Markets: Evidence from Vietnam", Journal of Management Studies, vol. 42, núm. 1, 2005, pp. 63-93.

Mody, Ashoka, Efraim Sadka y Assaf Razin, The Role of Information in Driving FDI Flows: Host-Country Transparency and Source-Country Specialization, Washington, DC, International Monetary Fund, 2003.

y Antu Panini Murshid, "Growing Up with Capital Flows", Journal of International Economics, vol. 65, núm. 1, 2005, pp. 249-266.

Mollick, Andre Varella, Rene Ramos-Duran y Esteban Silva-Ochoa, "Infrastructure and FDI Inflows into Mexico: A Panel Data Approach", Global Economy Journal, vol. 6, núm. 1, 2006, pp. 1-25.

Moran, Theodore H., Beyond Sweatshops, Washington, DC, Brookings Institution Press, 2002.

Mukim, Megha y Peter Nunnenkamp, "The Location Choices of Foreign Investors: A District-level Analysis in India”, The World Economy, vol. 35, núm. 7, 2012, pp. 886-918.

Pinto, Pablo M. y Santiago M. Pinto, "The Politics of Investment Partisanship and the Sectoral Allocation of Foreign Direct Investment”, Economics E̊ Politics, vol. 20, núm. 2, 2008, pp. 216-254.

—, Partisan Investment in the Global Economy: Why the Left Loves Foreign Direct Investment and FDI Loves the Left, Cambridge, MA, Cambridge University Press, 2013.

Przeworski, Adam, Michael E. Alvarez, José Antonio Cheibub, y Fernando Limongi, Democracy and Development. Political Institutions and Well-Being in the World, 1950-1990, Cambridge, MA, Cambridge University Press, 2000. 
Ramirez, Miguel D., "Are Foreign and Public Capital Productive in the Mexican Case? A Panel Unit Root and Panel Cointegration Analysis”, Eastern Economic Journal, vol. 36, núm. 1, 2010, pp. 70-87.

Romo, David, "Derramas tecnológicas de la inversión extranjera en la industria mexicana”, Comercio Exterior, vol. 53, núm. 3, 2003, pp. 230-243.

Samford, Steven y Priscila Ortega Gómez, "Subnational Politics and Foreign Direct Investment in Mexico", Review of International Political Economy, vol. 22, núm. 2, 2014, pp. 467-496.

Schiavon, Jorge, La Proyección Internacional de las Entidades Federativas: México ante el Mundo, Mexico, Secretaría de Relaciones Exteriores, 2006.

Secretaría de Economía, "Notas metodológicas sobre IED en México", en http:/ /www.2006-2012.economia.gob.mx/comunidad-negocios/inversion-extranjera-directa/estadistica-oficial-de-ied-en-mexico/notas-metodologicas

Waldkirch, Andreas, "The Effects of Foreign Direct Investment in Mexico since NAFTA", World Economy, vol. 33, núm. 5, 2010, pp. 710-745.

Zainal Abidin, Mahani y Nor Izzatina Abdul Aziz, "US Foreign Direct Investment in East Asia: Strategy and Policy Issues", Asian Economic Policy Review, vol. 4, núm. 2, 2009, pp. 248-266. 